\title{
Type I Interferons Modulate Vascular Function, Repair, Thrombosis, and Plaque Progression in Murine Models of Lupus and Atherosclerosis
}

\author{
Seth G. Thacker, ${ }^{1}$ Wenpu Zhao, ${ }^{1}$ Carolyne K. Smith, ${ }^{1}$ Wei Luo, ${ }^{1}$ Hui Wang, ${ }^{1}$ \\ Anuradha Vivekanandan-Giri, ${ }^{1}$ Bradley J. Rabquer, ${ }^{1}$ Alisa E. Koch, ${ }^{2}$ Subramaniam Pennathur, ${ }^{1}$ \\ Anne Davidson, ${ }^{3}$ Daniel T. Eitzman, ${ }^{1}$ and Mariana J. Kaplan ${ }^{1}$
}

Objective. Patients with systemic lupus erythematosus (SLE) have a notable increase in atherothrombotic cardiovascular disease (CVD) which is not explained by the Framingham risk equation. In vitro studies indicate that type I interferons (IFNs) may play prominent roles in increased CV risk in SLE. However, the in vivo relevance of these findings, with regard to the development of CVD, has not been characterized. This study was undertaken to examine the role of type I IFNs in endothelial dysfunction, aberrant vascular repair, and atherothrombosis in murine models of lupus and atherosclerosis.

Supported by the NIH (training grant T32-AI-007413, Public Health Service grant HL-088419 to Dr. Kaplan, and grant P30-AR48310 to the Rheumatic Disease Core Center, University of Michigan) and by the Anthony Gramer Fund in Inflammation Research (to Dr. Kaplan). Dr. Koch's work was supported by the Department of Veterans Affairs and the Frederick Huetwell and William Robinson, MD, Professorship in Rheumatology at the University of Michigan. Part of this work was conducted at the Chemistry Laboratory of the Michigan Diabetes Research and Training Center, University of Michigan Health System (funded by NIH grant P60-DK-20572) and the Molecular Phenotyping Core, Michigan Nutrition and Obesity Research Center, University of Michigan (funded by NIH grant P30-DK089503)

${ }^{1}$ Seth G. Thacker, PhD (current address: National Heart, Lung, and Blood Institute, NIH, Bethesda, Maryland), Wenpu Zhao, $\mathrm{MD}$, PhD, Carolyne K. Smith, BS, Wei Luo, MS, Hui Wang, MD, $\mathrm{PhD}$, Anuradha Vivekanandan-Giri, PhD, Bradley J. Rabquer, $\mathrm{PhD}$ (current address: Albion College, Albion, Michigan), Subramaniam Pennathur, MD, Daniel T. Eitzman, MD, Mariana J. Kaplan, MD: University of Michigan, Ann Arbor; ${ }^{2}$ Alisa E. Koch, MD: VA Medical Services, Ann Arbor, Michigan, and University of Michigan, Ann Arbor; ${ }^{3}$ Anne Davidson, MBBS: Feinstein Institute for Medical Research, Manhasset, New York.

Address correspondence to Mariana J. Kaplan, MD, Division of Rheumatology, University of Michigan, 5520 Medical Science Research Building-I, 1150 West Medical Center Drive, Ann Arbor, MI 48109-5680. E-mail: makaplan@umich.edu.

Submitted for publication October 25, 2011; accepted in revised form April 10, 2012.
Methods. Lupus-prone New Zealand mixed 2328 (NZM) mice and atherosclerosis-prone apolipoprotein Eknockout $\left(\operatorname{apoE}^{-/-}\right)$mice were compared to mice lacking type I IFN receptor (INZM and apoE ${ }^{-/-}$IFNAR $^{-/-}$ mice, respectively) with regard to endothelial vasodilatory function, endothelial progenitor cell (EPC) function, in vivo neoangiogenesis, plaque development, and occlusive thrombosis. Similar experiments were performed using NZM and apo $^{-/-}$mice exposed to an IFN $\alpha$-containing or empty adenovirus.

Results. Loss of type I IFN receptor signaling improved endothelium-dependent vasorelaxation, lipoprotein parameters, EPC numbers and function, and neoangiogenesis in lupus-prone mice, independent of disease activity or sex. Further, acute exposure to IFN $\alpha$ impaired endothelial vasorelaxation and EPC function in lupus-prone and non-lupus-prone mice. Decreased atherosclerosis severity and arterial inflammatory infiltrates and increased neoangiogenesis were observed in $\operatorname{apoE}^{-/-} \mathrm{IFNAR}^{-/-}$mice, compared to $\mathrm{apoE}^{-/-}$mice, while NZM and $\operatorname{apoE}^{-/}$mice exposed to IFN $\alpha$ developed accelerated thrombosis and platelet activation.

Conclusion. These results support the hypothesis that type I IFNs play key roles in the development of premature CVD in SLE and, potentially, in the general population, through pleiotropic deleterious effects on the vasculature.

Systemic lupus erythematosus (SLE) is associated with a dramatic increase in atherothrombotic cardiovascular disease (CVD), which is not explained by the Framingham risk equation (1-3). To date, no drug has been proven effective in decreasing CV risk in SLE. Therefore, it is crucial to establish the key drivers of vascular insult and atherosclerosis progression in lupus, 
in order to identify effective therapeutic and preventive targets.

While immune dysregulation may play a major role in premature CVD in SLE, the etiology of accelerated atherosclerosis in this disease remains unclear. Nevertheless, in vitro studies from our group and others indicate that type I interferons (IFNs) could play crucial roles in CVD development in SLE. This may be due to the capacity of IFN $\alpha$, and potentially other type I IFNs, to impair vascular repair (4), enhance foam cell formation (5), and activate platelets through changes in the megakaryocyte transcriptome (6). IFN $\beta$, another type I IFN, may promote atherosclerosis by promoting macrophage recruitment to arteries (7).

IFN $\alpha$ leads to an imbalance of vascular damage and repair by impairing the phenotype and function of bone marrow (BM)-derived endothelial progenitor cells (EPCs) (4) and by inducing transcriptional repression of angiogenic factors (8). However, while a prominent role for IFN $\alpha$ in lupus pathogenesis and clinical manifestations is supported by human and murine studies (9), it is unclear if type I IFNs are major inducers of vascular damage in vivo in SLE.

Increased endothelial cell (EC) damage in SLE is associated with perturbations in endothelium-dependent vasorelaxation (10), an established predictor of atherosclerosis progression (11). While most mouse strains (including lupus-prone mice) are resistant to dietinduced atherosclerosis (12), endothelial dysfunction develops in various models, including the lupus-prone $(\mathrm{NZB} \times \mathrm{NZW}) \mathrm{F}_{1}$ strain (13). Therefore, impairments in endothelium-dependent vasorelaxation can be used as a surrogate marker of vascular damage in murine models.

In this study, we demonstrate a prominent in vivo role of type I IFNs in the development of endothelial dysfunction, aberrant vascular repair, and atherothrombosis in murine models of lupus and atherosclerosis.

\section{MATERIALS AND METHODS}

Mice. Breeding pairs of lupus-prone NZM2328 (NZM) and NZM2328 IFN $\alpha \beta \mathrm{R}^{-1-}$ (INZM) mice were a gift from Dr. Chaim Jacob (University of Southern California, Los Angeles). Female mice were killed at 20 weeks of age (before proteinuria onset) or 30 weeks of age (after onset of significant proteinuria/active lupus). Male NZM and INZM mice were killed at 20, 30, or 40 weeks of age. Breeding pairs of IFN $\alpha \beta \mathrm{R}^{-1-}$ mice $(14,15)$ were a gift from Dr. H. W. Virgin (Washington University, St. Louis, MO). The apoE $\mathrm{E}^{-1-}$ IFN $\alpha \beta \mathrm{R}^{-1-}$ mice were generated by backcrossing apoE ${ }^{-1-}$ mice (The Jackson Laboratory) with IFN $\alpha \beta \mathrm{R}^{-1-}$ mice, both on the C57BL/6 background, to generate double heterozygotes, followed by backcrossing the offspring back to the apoE $\mathrm{E}^{-1-}$ background. The genotype was confirmed by polymerase chain reaction. Protocols were approved by the University of Michigan's Committee on Use and Care of Animals. Mice were bred and housed in a specific pathogenfree barrier facility at the University of Michigan. Proteinuria was assessed using Uristix 4 (Siemens).

Adenovirus administration. Murine IFN $\alpha$-expressing adenovirus (AdIFN $\alpha$ ) was synthesized as previously described (16). Empty adenovirus control (AdControl) was obtained through the University of Michigan's Vector Core. Adenovirus was administered to 12-week-old NZM and BALB/c mice and to 8 -week-old apoE $\mathrm{E}^{-1-}$ mice, at $3-4 \times 10^{9}$ particles in $100 \mu \mathrm{l}$ phosphate buffered saline (PBS), via tail vein injection. Injection efficacy was assessed by spleen size and levels of type I IFN-regulated genes (Mx1, Mcp1, Irf7, Ip10, and Isg15) in spleen and BM (data not shown), since IFN $\alpha$ levels were not reliably detected in the serum by enzyme-linked immunosorbent assay (ELISA), as previously reported $(16,17)$.

Assessment of endothelium-dependent vasorelaxation. Endothelium-dependent vasorelaxation was assessed as previously described by our group $(13,18)$. Mouse aortas were cut into 2-mm-long rings, which were mounted in a myograph system (DMT-USA). Concentration-response curves were obtained after contracting vessels with phenylephrine $\left(10^{-6}\right.$ moles/liter), followed by acetylcholine (ACh; $10^{-7}$ moles/liter) to test endothelial integrity. Vessels were recontracted with phenylephrine at $75 \%$ maximum response concentration $\left(\mathrm{EC}_{75}\right)$ and allowed to reach a stable plateau in the contraction. ACh $\left(10^{-10}-10^{-5}\right.$ moles/liter $)$ was added cumulatively to examine endothelium-dependent relaxation, expressed as percent phenylephrine $\mathrm{EC}_{75}$ contraction (18).

Quantification of EPC number and differentiation. Mononuclear fractions from BM and spleen single-cell suspensions or from whole blood were obtained by Histopaque 1083 (Sigma) gradient centrifugation. Red blood cells (RBCs) were lysed with RBC lysis buffer (BioLegend). Approximately 1-5 $\times$ $10^{6} \mathrm{BM}$ and spleen cells or $2-5 \times 10^{5}$ peripheral blood mononuclear cells were incubated with monoclonal antibodies against murine CD34, vascular endothelial growth factor receptor 2 (VEGFR-2), Sca-1, CD117, CD3, CD19, and CD45 (all from eBioscience) and murine lineage depletion cocktail (Miltenyi Biotec) for 30 minutes on ice, followed by incubation with Pacific Blue-labeled annexin V (BioLegend). Fluorescence-activated cell sorting (FACS) was performed with FACSCalibur (BD Biosciences) or CyAn (Beckman), followed by analysis with FlowJo (Tree Star). EPCs were quantified as CD34+VEGFR-2+Lin- or Sca-1+CD117+Lin-, as previously described $(13,19,20)$.

$\mathrm{BM}$ or spleen mononuclear cell fractions were plated onto fibronectin-coated plates $\left(1 \times 10^{6}\right.$ cells $\left./ \mathrm{cm}^{2}\right)$ in EGM-2 BulletKit media (Lonza) with 5\% heat-inactivated fetal bovine serum. On day 7, cells were incubated with fluorescein isothiocyanate (FITC)-conjugated Bandeiraea (Griffonia) simplicifolia lectin 1 (BS-1; Vector) and Dil-labeled acetylated lowdensity lipoprotein (LDL; Biomedical Technologies) for 4 hours, then analyzed by fluorescence microscopy (Olympus IX70). Images were acquired using a CoolSNAP HQ2 monochrome camera (Photometrics) and MetaMorph Premier version 6.3 acquisition software (Molecular Devices). Final processing was performed with Adobe Photoshop CS2. Cells costaining with BS-1 and acetylated LDL were considered to 
be mature ECs and were quantified in 5-6 random fields per well using CellC software.

EPCs were quantified in mouse aorta sections using rabbit anti-mouse CD31 (1:20), rat anti-mouse Sca-1 (1:200) (both from Abcam), and appropriate isotype controls (eBioscience). Secondary antibodies included Alexa Fluor 488conjugated goat anti-rat IgG and Alexa Fluor 555-conjugated donkey anti-rabbit IgG (both from Invitrogen). Nuclei were counterstained by DAPI (Invitrogen). Images were acquired with an Olympus BX51A fluorescence microscope.

In vivo Matrigel plug angiogenesis assay. Matrigel plug assays were performed as previously described (21). Growth factor-reduced Matrigel (500 $\mu \mathrm{l}$; Becton Dickinson) containing $20 \mathrm{n} M$ basic fibroblast growth factor (R\&D Systems) was injected subcutaneously, and mice were killed 7 days postinjection. Plugs were excised, and angiogenesis was measured using the 3,3',5,5'-tetramethylbenzidine method (22) to quantify hemoglobin, standardized to plug weight, as an indirect measure of new blood vessel formation. In some experiments, a portion of the plug was frozen in OCT medium and stained with polyclonal rabbit anti-von Willebrand factor (DakoCytomation) or rabbit IgG, followed by FITC-conjugated anti-rabbit IgG (Invitrogen). Nuclear staining was performed with DAPI. Images were acquired with an Olympus fluorescence microscope.

Carotid arterial thrombosis. Mice were subjected to photochemical injury of the right carotid artery by rose bengal dye, as previously described (23). Mice were anesthetized and placed under a dissecting microscope (Nikon SMZ2-T; Mager Scientific), and the right carotid artery was isolated. A Doppler flow probe (Transonic) was applied, and rose bengal dye (Fisher Scientific) diluted in PBS was injected into the tail vein (50 mg/kg). A $1.5-\mathrm{mW}$ green light laser $(540 \mathrm{~nm})$ (Melles Griot) was applied to the desired site of injury, from a distance of $6 \mathrm{~cm}$, and vessel flow was monitored until occlusive thrombosis occurred, defined as flow cessation for at least 10 minutes.

Platelet activation assessment. Serum soluble P-selectin level was quantified by ELISA (R\&D Systems). Leukocyteplatelet aggregates (Gr-1+CD61+ cells) and platelet P-selectin level were quantified by FACS (CyAn), after $50 \mu \mathrm{l}$ of blood was added to $100 \mu$ l of heparin (10,000 IU/ml; Sigma), and incubated with fluorochrome-conjugated antibodies against murine CD61, CD62P, and Gr-1 (all from eBioscience), followed by RBC lysis and analysis by FlowJo.

Atherosclerosis quantification and immunohistochemical analysis of mouse aortic valves. Animals were fed a Western diet (Harlan) for $\sim 10$ weeks, starting at 8-10 weeks of age. At euthanasia, mice were perfused with saline, carcasses were fixed in formalin, and arterial trees were dissected and placed in $70 \%$ ethanol, then stained with oil red O (Sigma). The surface area occupied by atherosclerosis was quantified at the aortic arch and major branches using Image-Pro Plus software (Media Cybernetics) and expressed as percentage of total surface area examined, as previously described (24).

Rat anti-mouse F4/80 (1:200) and rabbit anti-mouse CD3 (1:100) (both from Abcam) staining was followed by peroxidase-conjugated AffiniPure goat anti-rat $\operatorname{IgG}(\mathrm{H}+\mathrm{L})$ or peroxidase-conjugated donkey anti-rabbit $\mathrm{IgG}$, respectively (1:200; Jackson ImmunoResearch). Images were visualized using a DAB-Plus Substrate Kit (Invitrogen). Digital images were obtained with a DP71 camera mounted on an Olympus BX51B microscope. Quantification of F4/80 and CD3 staining area was performed using ImageJ software (NIH Image, National Institutes of Health; online at http://rsbweb.nih.gov/ $\mathrm{ij} /$ ).

High-density lipoprotein (HDL) oxidation assessment. HDL was isolated from mouse plasma by buoyant density ultracentrifugation as previously described (25). HDL protein was precipitated with ice-cold trichloroacetic acid and delipidated with water/methanol/water-washed diethyl ether. The protein pellet was subjected to acid hydrolysis using $4 \mathrm{~N}$ methane sulfonic acid supplemented with $1 \%$ benzoic acid at $110^{\circ} \mathrm{C}$ for 24 hours, after addition of isotopically labeled internal standards. Amino acids were isolated from hydrolysate with Supelclean ENVI-Chrom P columns (Supelco) as previously described (26). Liquid chromatography-electrospray ionization tandem mass spectrometry using multiple reaction monitoring (27) was used to quantify 3-nitrotyrosine. The ratio of peak areas of the analyte multiple reaction monitoring transitions versus corresponding isotopically labeled internal standard was used to calculate 3-nitrotyrosine and tyrosine content. Results were normalized to tyrosine protein content, the precursor of 3-nitrotyrosine.

Statistical analysis. Results are presented as the mean \pm SEM. Statistical analysis was performed using Student's $t$-test, and data were analyzed using GraphPad Prism software version 5. The median effective concentration $\left(\mathrm{EC}_{75}\right)$ for agent-induced vasorelaxation was calculated by nonlinear regression analysis (GraphPad Prism version 5).

\section{RESULTS}

Improved endothelial vasorelaxation and EPC numbers in the absence of type I IFN signaling in lupus-prone NZM mice. We previously reported that female lupus-prone NZB $\times$ NZW mice have impaired endothelial and EPC function, and decreased tissue and circulating EPCs. This impairment correlates with enhanced expression of type I IFN-responsive genes in EPC compartments (13). To directly address the putative role of type I IFNs in EC and EPC function in lupus, we examined NZM mice, which show similar lupus disease progression to the NZB $\times$ NZW strain from which they were derived (28). NZM mice were compared to NZM mice lacking type I IFN receptor (INZM mice). When the endothelium-dependent response of thoracic aortas to ACh was quantified, 30-week-old female INZM mice displayed significant improvements in vasorelaxation compared to age- and sex-matched NZM mice (Figure 1A).

Compared to female NZM mice, 30-week old female INZM mice displayed increased numbers of BM and circulating EPCs (Figure 1B). (Additional results are available online at http://deepblue.lib.umich.edu/.) Younger (20-week-old) INZM female mice displayed 

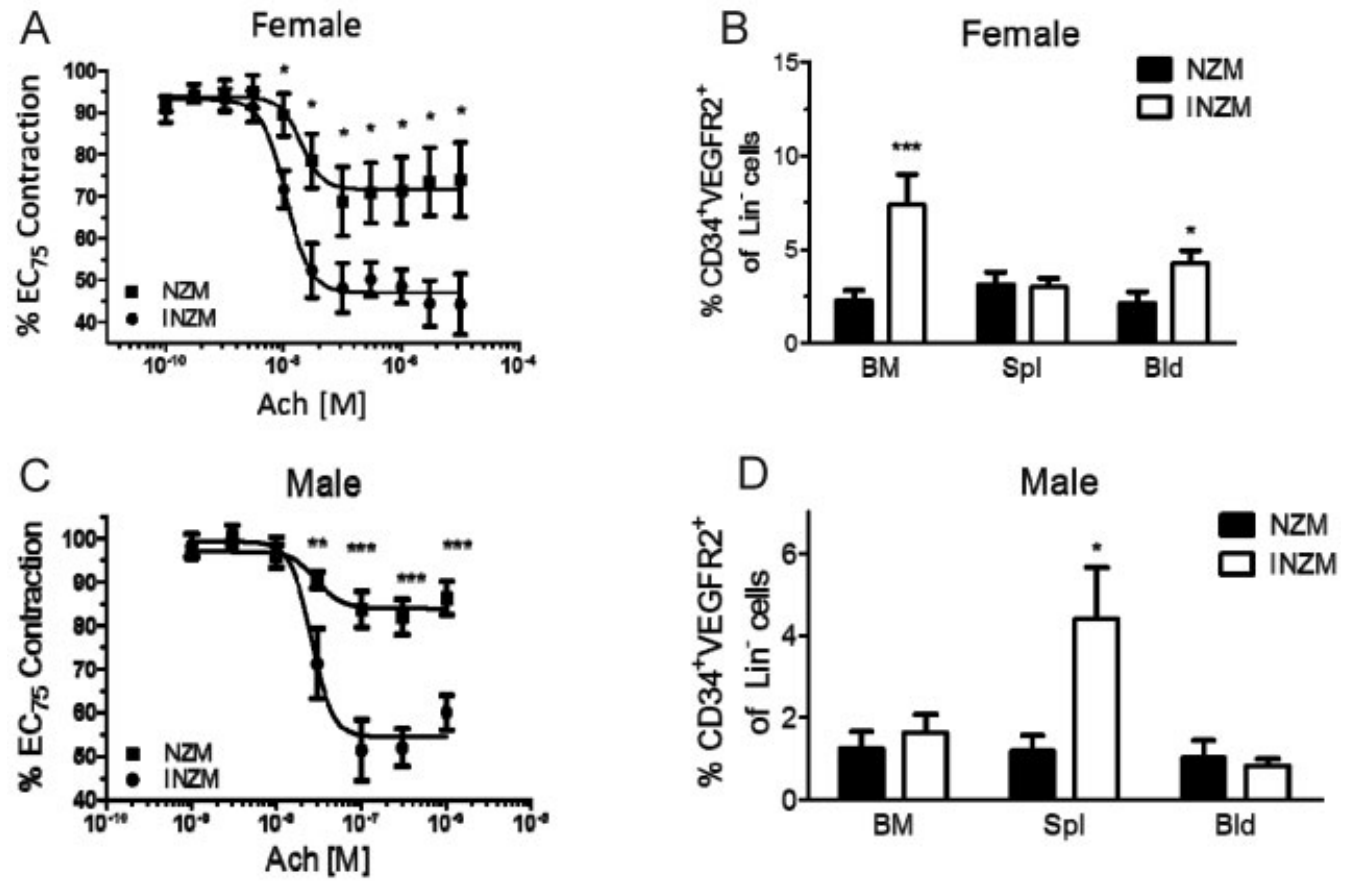

Figure 1. Improved endothelial function and increased endothelial progenitor cell (EPC) numbers in the absence of type I interferon signaling in lupus-prone NZM2328 (NZM) mice. A, Increased aortic endothelium-dependent vasorelaxation in response to acetylcholine (ACh) in 30-week-old female NZM IFNAR ${ }^{-1-}$ (INZM) mice compared to age- and sex-matched NZM mice. B, Increased numbers of bone marrow (BM) and blood (Bld) EPCs in 30-week-old female INZM mice compared to age- and sex-matched NZM mice. C, Improved endothelium-dependent vasorelaxation in 40-week-old male INZM mice compared to age and sex-matched NZM mice. D, Increased numbers of spleen (Spl) EPCs in 40-week-old male INZM mice compared to age- and sex-matched NZM mice. Values are the mean \pm SEM $(n=8$ mice per group in A, 13 mice per group in $\mathbf{B}$, and 6 mice per group in $\mathbf{C}$ and $\mathbf{D})$. $*=P<0.05 ; * *=P<0.01 ; * * *=P<0.001$, versus INZM mice in $\mathbf{A}$ and $\mathbf{C}$ and versus NZM mice in $\mathbf{B}$ and $\mathbf{D}$. EC $75=$ $75 \%$ maximum response concentration.

increases in spleen EPC numbers compared to NZM mice $(P<0.05$; data not shown), while no changes were observed in their BM or blood EPC numbers. In contrast to our previous findings in NZB $\times$ NZW mice (13), we did not observe differences in EPC apoptosis upon loss of type I IFN signaling in female NZM mice (data not shown).

The beneficial effects on endothelial function and EPC numbers observed in INZM mice could be a direct result of blocking type I IFN signaling or secondary to abrogation of lupus development, including renal disease, in INZM mice (29). To determine if type I IFNs directly impair vasorelaxation in murine models of lupus, independent of disease activity, we performed similar experiments in male NZM and INZM mice, since neither of them develop significant SLE features. Similar to autologous female mice, 40-week-old male INZM mice demonstrated significant increases in endotheliumdependent vasorelaxation and spleen EPC numbers compared to age- and sex-matched NZM mice (Figures $1 \mathrm{C}$ and D), with no changes in EPC apoptosis. There were no significant differences between the male groups at younger ages (20 weeks and 30 weeks of age; data not shown). These results indicate that type I IFNs significantly modulate endothelium-dependent vasorelaxation and EPC numbers in lupus-prone mice, independently of disease activity or sex.

Improved EPC function and in vivo neoangiogenesis in the absence of type I IFN signaling in lupusprone NZM mice. We previously reported that EPCs from NZB $\times$ NZW mice and lupus patients display decreased capacity to differentiate into mature ECs when exposed to proangiogenic stimulation $(4,13)$. Confirming a role for type I IFNs in impairing endothelial differentiation, EPCs from female INZM mice displayed increased ability to differentiate into mature ECs when compared to those from age-matched female NZM mice. This improvement was observed by 20 weeks of age, before evidence of active lupus, and was maintained after florid disease development at 30 weeks of age (Figure 2A). These findings were confirmed in male mice, as BM EPCs from 40-week old (but not 30-week 
A

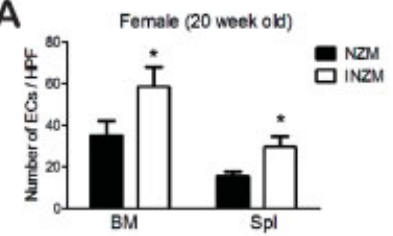

B

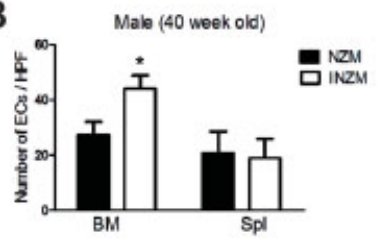

D

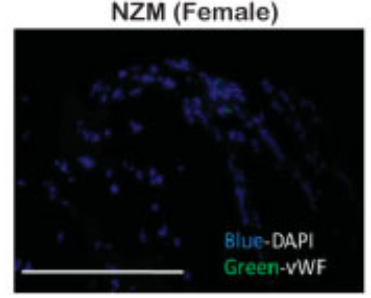

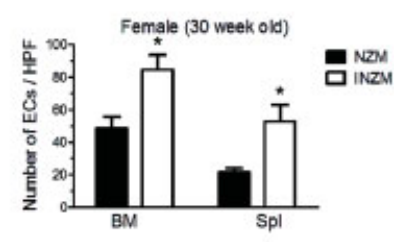

C

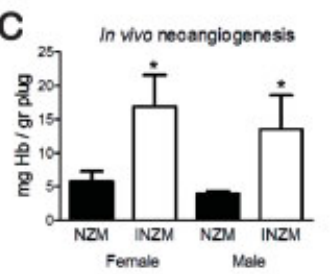

INZM (Female)

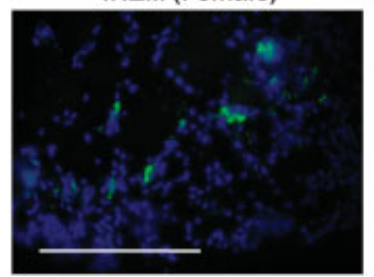

Figure 2. Improved EPC function and in vivo neoangiogenesis in the absence of type I interferon signaling in lupus-prone NZM mice. A, Increased capacity of EPCs to differentiate into mature endothelial cells (ECs) in female INZM mice at 20 weeks of age (left) and at 30 weeks of age (right) compared to age- and sex-matched NZM mice. B, Significant improvement in BM EPC differentiation into mature ECs in male INZM mice at 40 weeks of age. Values in $\mathbf{A}$ and $\mathbf{B}$ are the mean $\pm \operatorname{SEM}(\mathrm{n}=6-8$ mice per group in $\mathbf{A}$ and 9 mice per group in $\mathbf{B})$. $*=P<0.05$ versus NZM mice. hpf $=$ high-power field. $\mathbf{C}$, Enhanced in vivo neoangiogenesis in 25-30-week-old female and 30-40-week-old male INZM mice compared to age- and sex-matched NZM mice, as assessed by total $\mathrm{mg}$ hemoglobin $(\mathrm{Hb})$ per gram of subcutaneous Matrigel plug. Values are the mean \pm SEM $(n=16$ female mice per group and 14 male mice per group). $*=P<0.05$ versus NZM mice. D, Representative images of Matrigel plugs demonstrating increased numbers of von Willebrand factor (vWF)-positive ECs in 25-30-weekold female INZM mice compared to age- and sex-matched NZM mice ( $\mathrm{n}=3$ mice per group). There was an $\sim 7$-fold increase in vWFpositive cells in female INZM mice compared to those of female NZM mice, when adjusted for DAPI-positive cells. Bar $=200 \mu \mathrm{m}$; original magnification $\times 40$. See Figure 1 for other definitions.

old) INZM mice displayed significant increases in differentiation into mature ECs compared to cells from age-matched NZM mice (Figure 2B and data not shown).

In vivo Matrigel plug assays were performed to assess the effects of type I IFNs on the capacity of lupus-prone mice to form new blood vessels. Compared to age- and sex-matched NZM mice, female INZM mice (25-30 weeks old) and male INZM mice (30-40 weeks old) displayed significant increases in in vivo neoangiogenesis (Figures $2 \mathrm{C}$ and $\mathrm{D}$ ). These results demonstrate that type I IFNs promote decreased angiogenesis in lupus-prone mice, independent of disease activity or sex.
Worsening of endothelium-dependent vasorelaxation and EPC differentiation after acute exposure to IFN $\boldsymbol{\alpha}$ in murine lupus. Since lack of type I IFN signaling significantly improved endothelial and EPC function in lupus-prone mice, we explored whether acute exposure to enhanced levels of IFN $\alpha$, such as those seen in acute infections (30) or lupus flares (31), promoted the opposite effects. Compared to mice that received AdControl, female NZM mice that received systemic $\operatorname{AdIFN} \alpha$ at 12 weeks of age displayed significant decreases in endothelium-dependent vasorelaxation by 3 weeks postinjection. AdIFN $\alpha$ administration did not impact EPC numbers or apoptosis (data not shown). However, EPC differentiation into mature ECs was significantly reduced in mice that received $\operatorname{AdIFN} \alpha$ compared to those that received AdControl (Figure 3A).

Similar to the NZB $\times$ NZW parental strain (16), AdIFN $\alpha$ administration led to acceleration of nephritis onset. (Details on the clinical and demographic characteristics of the mouse strains are available online at http://deepblue.lib.umich.edu/.) To establish whether the endothelial defects observed following AdIFN $\alpha$ administration were due to lupus exacerbation, similar experiments were performed in male NZM mice, since male mice do not develop lupus following IFN $\alpha$ administration. Three weeks after AdIFN $\alpha$ injection, 15-weekold male NZM mice also exhibited significantly decreased endothelium-dependent vasorelaxation. As in female NZM mice, AdIFN $\alpha$ did not modify EPC numbers or apoptosis. In contrast to female NZM mice, male mice did not display impaired EPC differentiation following AdIFN $\alpha$ exposure (Figure 3B). These findings indicate that most of the acute effects of IFN $\alpha$ on the vasculature are independent of lupus disease activity or sex.

To assess whether the deleterious effects of IFN $\alpha$ on EC and EPC function are dependent on a lupusprone genetic background, AdIFN $\alpha$ or AdControl was administered to 12-week-old female BALB/c mice. While these mice do not develop SLE upon exposure to type I IFNs, they developed decreased endotheliumdependent vasorelaxation and impaired EPC differentiation (Figure 3C), with no modifications in EPC numbers or apoptosis (data not shown). These results demonstrate that type I IFNs can promote endothelial and EPC dysfunction independent of autoimmune background.

Modulation of plaque severity and arterial inflammation by type I IFNs in murine models of atherosclerosis. Endothelial dysfunction predicts plaque development (11), and IFN $\alpha$ can induce foam cell formation in vitro (5). Further, myeloid IFN $\beta$ signaling promotes 
A

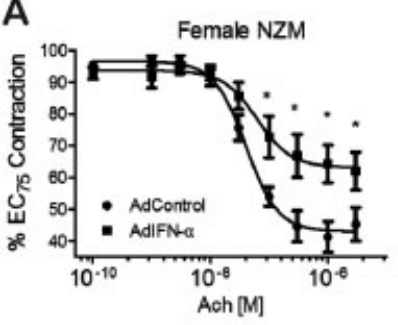

B
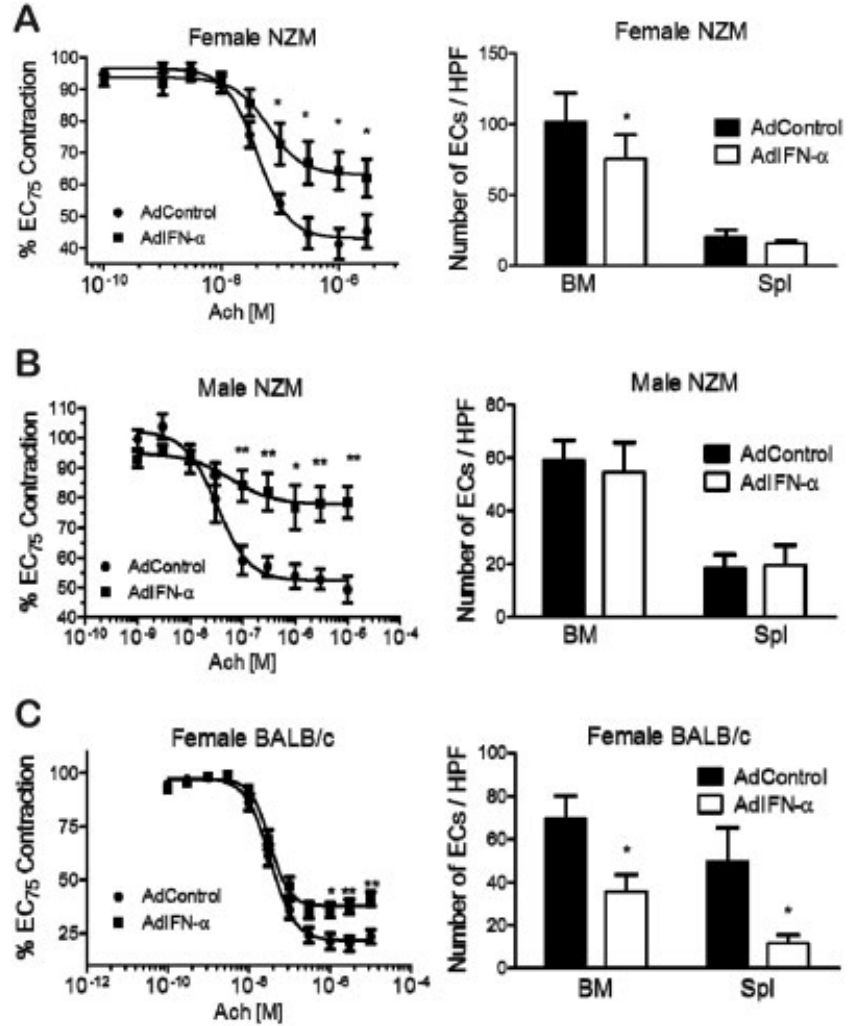

Figure 3. Impairment of endothelium-dependent vasorelaxation and EPC function in lupus-prone and non-lupus-prone mice after acute exposure to interferon- $\alpha$ (IFN $\alpha)$. A, Decreased endothelium-dependent vasorelaxation (left) and reduced EPC differentiation (right) 3 weeks postinjection in female NZM mice exposed to adenovirus IFN $\alpha$ $(\operatorname{AdIFN} \alpha)$ at 12 weeks of age, compared to age- and sex-matched NZM mice exposed to adenovirus control (AdControl). B, Decreased endothelium-dependent vasorelaxation (left) and unaltered EPC function (right) in male NZM mice exposed to AdIFN $\alpha$ compared to mice exposed to AdControl. C, Decreased endothelium-dependent vasorelaxation (left) and reduced EPC differentiation (right) 3 weeks postinjection in female non-lupus-prone $\mathrm{BALB} / \mathrm{c}$ mice exposed to AdIFN $\alpha$ at 12 weeks of age. Values are the mean \pm SEM $(n=10-11$ mice per group in $\mathbf{A}, 5-8$ mice per group in $\mathbf{B}$, and 12 mice per group in $\mathbf{C}$ ). $*=P<0.05$; $* *=P<0.01$, versus mice exposed to AdControl. $\mathrm{ECs}=$ endothelial cells; hpf $=$ high-power field (see Figure 1 for other definitions).

atherosclerosis by stimulating macrophage recruitment to arteries (7). Since NZB $\times$ NZW and NZM mice are resistant to developing florid diet-induced atherosclerosis, we investigated whether IFN $\alpha$ directly affected plaque severity in vivo in atherosclerosis-prone apo $\mathrm{E}^{-/-}$ mice (32). This was tested by exposing mice to acute AdIFN $\alpha$ infection at the time of Western diet initiation, and through genetic abrogation of type I IFN signaling (apoE $\left.{ }^{-1-} \mathrm{IFNAR}^{-1-}\right)$. There were no significant differences in atherosclerosis severity between male and female mice at the various time points examined. After 10 weeks on a Western diet, female and male apoE ${ }^{-1-}$ IFNAR $^{-1-}$ mice (18-20 weeks old) showed significantly reduced atherosclerotic lesion size when compared to age- and sex-matched apoE ${ }^{-1-}$ mice (Figures $4 \mathrm{~A}$ and $\mathrm{B}$ ). Additionally, there was a close to significant trend toward larger atherosclerotic lesions in apoE ${ }^{-1-}$ mice receiving AdIFN $\alpha$ compared to apoE ${ }^{-1-}$ mice receiving AdControl $(P=0.0604)$ (Figure 4A).

Aortic valve sections were stained with macrophage- and T cell-specific antibodies. Less severe atherosclerosis in apoE ${ }^{-1-}$ IFNAR $^{-1-}$ mice was associated with significant decreases in macrophage and $\mathrm{T}$ cell vasculature infiltration compared to apoE $\mathrm{E}^{-1-}$ mice (Figure 4C). (Additional results are available online at http://deepblue.lib.umich.edu/.) These findings indicate that type I IFNs modulate atherosclerosis severity and arterial inflammatory infiltrates. Aberrant vascular repair may play a role in atherosclerosis development in $a p o E^{-1-}$ mice (33). Compared to $a p o E^{-/-}$mice, apoE ${ }^{-/-} \mathrm{IFNAR}^{-/-}$mice displayed increased angiogenic capacity, as determined by an in vivo Matrigel plug assay, and increased numbers of arterial wall EPCs (Figure 5). These results indicate that lack of type I IFN signaling promotes enhanced neoangiogenesis in atherosclerosis-prone mice.

Type I IFNs may modulate HDL oxidation in murine lupus. HDL oxidation renders the lipoprotein dysfunctional and proinflammatory and may contribute to atherogenesis. Lupus patients have increased plasma levels of oxidized HDL $(34,35)$. Previous work showed that HDL nitrotyrosine content, a specific oxidative process mediated by reactive nitrogen species, is elevated in humans with established CVD (26). To assess the contribution of type I IFNs to HDL nitration, HDL was isolated from murine plasma and its nitrotyrosine content was quantified. Thirty-week-old female NZM mice had notable elevations in HDL nitrotyrosine content compared with age- and sex-matched BALB/c mice, and this increase was abrogated in female INZM mice. In contrast, AdIFN $\alpha$ administration did not significantly affect HDL nitrotyrosine content in $\mathrm{NZM}$, apoE ${ }^{-1-}$, or BALB/c mice (Figure 5). Significant differences in HDL nitrotyrosine content were not observed between apoE ${ }^{-1-}$ IFNAR $^{-1-}$ mice and apoE ${ }^{-/-}$mice (mean \pm SEM $688 \pm 83$ versus $613 \pm 70 \mu$ moles/mole tyrosine; $\mathrm{n}=5-8$ samples per group).

Modulation of thrombosis and platelet activation by type I IFNs in murine models of lupus and atherosclerosis. Atheroma formation with superimposed thrombosis represents the pathophysiologic process in 
A

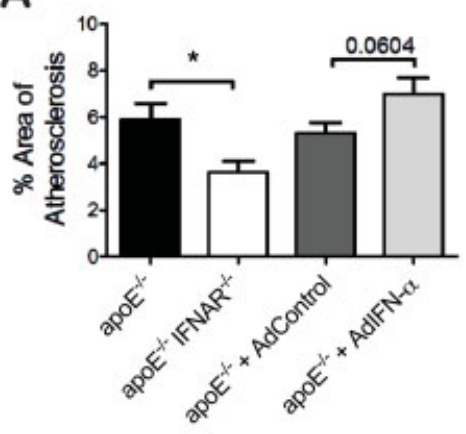

B
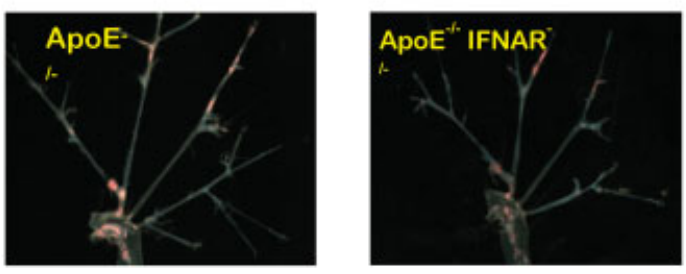

C
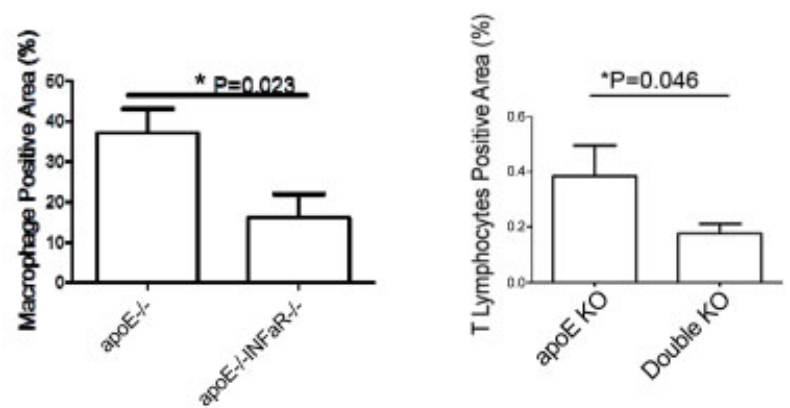

Figure 4. Atherosclerosis severity and inflammatory arterial infiltration are sensitive to perturbations in type I interferon (IFN) signaling in apoE $\mathrm{E}^{-1-}$ mice. A, Significantly reduced atherosclerotic lesion size in 18-20-week-old apoE ${ }^{-/}$IFNAR $^{-/-}$mice compared to age- and sex-matched apoE $\mathrm{E}^{-/-}$mice $(\mathrm{n}=13$ mice per group). No differences in atherosclerosis severity were observed between female and male mice ( $\mathrm{n}=9$ mice per group). The apoE $\mathrm{E}^{-/-}$mice that received adenovirus IFN $\alpha(\operatorname{AdIFN} \alpha)$ at 12 weeks of age displayed a trend toward increased lesion size at 20 weeks of age $(n=13$ mice per group). Values are the mean \pm SEM. $*=P<0.05$. B, Representative en face views of oil red O-stained aortic trees from an $a p o E^{-/-}$mouse and an apoE ${ }^{-/-}$IFNAR $^{-/-}$mouse, demonstrating decreased lesion size in apoE $\mathrm{E}^{-/-}$mice lacking type I IFN receptor. The percentages of atherosclerotic lesions in these representative images were $25.8 \%$ for the apoE $\mathrm{E}^{-1-}$ mouse and $14.1 \%$ for the apoE ${ }^{-1-} \mathrm{IFNAR}^{-1-}$ mouse. Original magnification $\times 10$. C, Significantly reduced macrophage and $\mathrm{T}$ cell infiltration of aortic valves in 18-20-week-old apoE ${ }^{-/-}$IFNAR $^{-1-}$ double-knockout (KO) mice compared to apoE ${ }^{-/-}$knockout mice. Values are the mean \pm SEM percent of area infiltrated by macrophages or T cells, after counting 6 random fields $(\mathrm{n}=6$ mice per group).

acute coronary syndromes (ACS). SLE patients are at increased risk of thrombotic events, and this phenome- non may be multifactorial. Type I IFNs can affect the megakaryocyte transcriptome and induce platelet activation in vitro (6). To address whether type I IFNs promote a prothrombotic environment in vivo, a model of carotid clot induction following photochemical injury was used. After AdIFN $\alpha$ exposure, apoE ${ }^{-/-}$and NZM

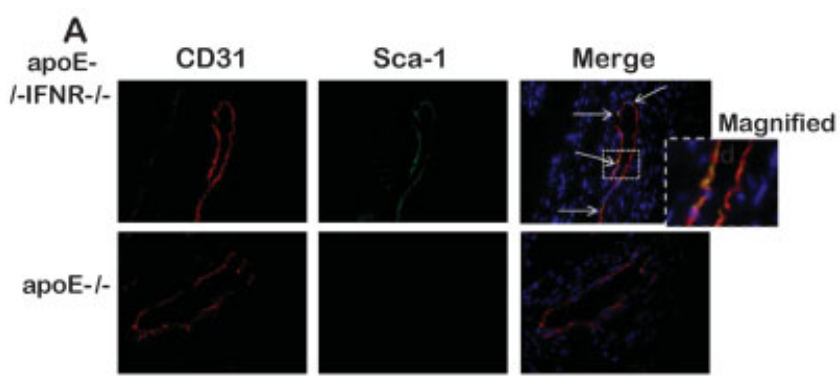

B

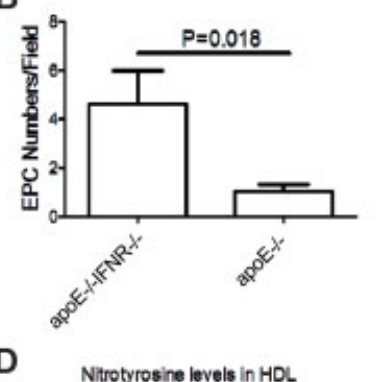

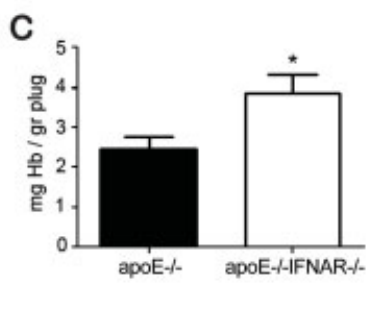

Figure 5. Modulation of neoangiogenesis and high-density lipoprotein (HDL) oxidation by type I interferon (IFN) signaling. $\mathbf{A}$ and B, Representative images (A) and quantification (B) of cells costaining for Sca-1 and CD31 in the aortic roots of apoE ${ }^{-/-} \mathrm{IFNAR}^{-/-}$and apoE ${ }^{-1-}$ mice. Sca-1 staining was graded according to intensity on a $0-2+$ scale, where $0=$ no staining and $2+=$ maximum intensity staining. Cells showing maximum intensity staining were counted in 3 different representative fields per slide. In $\mathbf{A}$, the boxed area shows a higher-magnification view of the outlined region, displaying Sca-1+ $\mathrm{CD} 31+$ cells in the apoE $\mathrm{E}^{-/-} \mathrm{IFNAR}^{-/-}$mouse. Original magnification $\times 400$. Values in $\mathbf{B}$ are the mean \pm SEM number of doublepositive cells ( $n=5$ mice per group). $\mathbf{C}$, Increased neoangiogenesis in 20-26-week-old apoE ${ }^{-1-}$ IFNAR $^{-1-}$ mice compared to age- and sexmatched apoE ${ }^{-1-}$ mice. Neoangiogenesis was assessed as mg hemoglobin $(\mathrm{Hb})$ per gram Matrigel plug. Values are the mean $\pm \operatorname{SEM}(\mathrm{n}=$ 11 mice per group). $*=P<0.05$ versus apoE $\mathrm{E}^{-/-}$mice. $\mathbf{D}$, Increased levels of proinflammatory HDL, which decrease significantly in the absence of type I IFN signaling, in 30-week-old female lupus-prone NZM mice. Values are the mean \pm SEM micromoles HDL nitrotyrosine per mole tyrosine $(\mathrm{n}=8-10$ mice per group). $* *=P<0.01$. See Figure 1 for other definitions. 
A

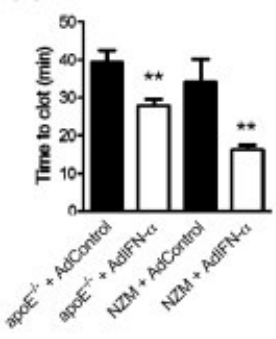

B

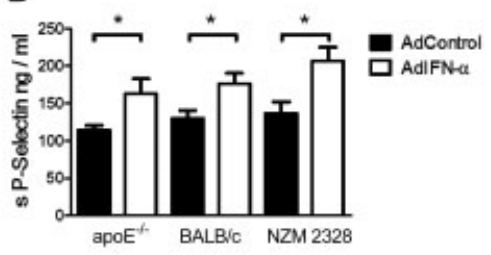

C

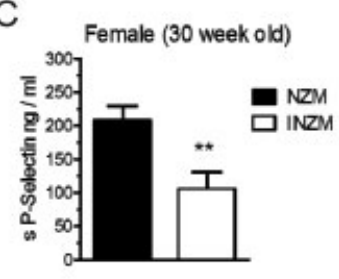

D
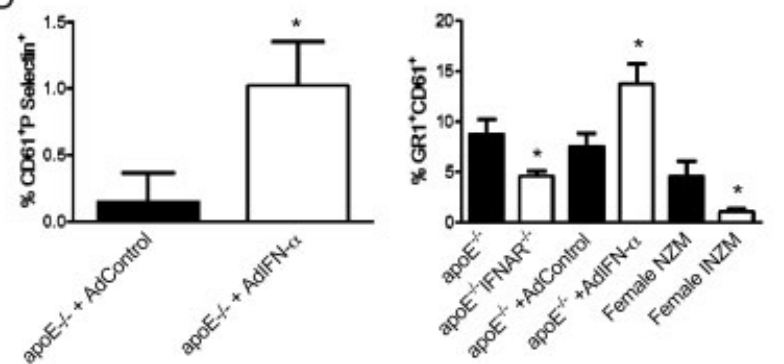

Figure 6. Modulation of thrombosis, endothelial activation, and platelet activation by type I interferons (IFNs) in atherosclerosis-prone and lupus-prone mice. A, Significantly shorter time to occlusive thrombosis in the mid common carotid artery in 10-14-week-old apoE $\mathrm{E}^{-/-}$and NZM mice exposed to adenovirus $\operatorname{IFN} \alpha(\operatorname{AdIFN} \alpha)$ compared to age- and sex-matched mice exposed to AdControl (n = 10-14 mice per group). Lack of type I IFN signaling in these mice (20-22 weeks old) had no effect on time to occlusive thrombosis ( $\mathrm{n}=$ 10-14 mice per group). $* *=P<0.01$ versus mice exposed to AdControl. B, Correlation of shorter time to occlusive thrombosis with increased serum soluble P-selectin (sP-selectin) levels in 10-11-weekold apoE $\mathrm{E}^{-1-}$ mice $(\mathrm{n}=8$ mice per group) and 14-week-old NZM mice ( $\mathrm{n}=6$ mice per group). AdIFN $\alpha$ also increased serum P-selectin levels in 17-week-old BALB/c mice ( $\mathrm{n}=6$ mice per group). $*=P<0.05$. C, Significantly decreased serum P-selectin levels in 30-week-old INZM mice compared to NZM mice ( $\mathrm{n}=10-15$ mice per group). $* *=P<0.01$ versus NZM mice. $\mathbf{D}$, Modulation of platelet activation by type I IFNs, as assessed by P-selectin expression on platelets ( $\mathrm{n}=$ 9-10 mice per group) (left) and the percentage of leukocyte-platelet aggregates ( $\mathrm{n}=11-16$ mice per group) (right) in 20-25-week old mice. $*=P<0.05$ versus wild-type mice or mice exposed to AdControl. Values are the mean \pm SEM. See Figure 1 for other definitions.

mice displayed significantly shorter time to occlusive thrombosis in the mid common carotid artery compared to AdControl-treated mice (Figure 6A). In contrast, no significant differences were observed with regard to time to occlusive thrombosis between $a p o E^{-/-}$mice and apoE $^{-/-}$IFNAR $^{-1-}$ mice or between NZM mice and INZM mice (mean \pm SEM $29.1 \pm 2.3$ versus $31.2 \pm 3.2$ minutes and $20 \pm 2.4$ versus $26.2 \pm 4.6$ minutes, respectively).

Accelerated thrombosis in AdIFN $\alpha$-treated apoE $\mathrm{E}^{-1-}$ mice and NZM mice was associated with enhanced endothelial and platelet activation, assessed by serum P-selectin levels. Similar increases in soluble P-selectin levels following $\operatorname{AdIFN} \alpha$ exposure were detected in female BALB/c mice (Figure 6B). Compared to age- and sex-matched NZM mice, soluble P-selectin levels were decreased in 30-week-old female INZM mice (Figure 6C). Administration of $\operatorname{AdIFN} \alpha$ to apoE $\mathrm{E}^{-/-}$ mice and NZM mice resulted in enhanced platelet $\mathrm{P}$-selectin levels and an increased percentage of leukocyte-platelet aggregates. Conversely, INZM mice had significant decreases in the percentage of leukocyteplatelet aggregates compared to NZM mice (Figure 6D). Compared to apoE ${ }^{-1-}$ mice, apoE ${ }^{-1-} \mathrm{IFNAR}^{-1-}$ mice exhibited a decreased percentage of leukocyteplatelet aggregates (Figure 6E) but no significant changes in platelet P-selectin levels (data not shown). These results demonstrate that type I IFNs accelerate thrombosis and induce platelet activation in murine models of lupus and atherosclerosis.

\section{DISCUSSION}

Broad activation of type I IFN pathways in SLE may play crucial roles in disease pathogenesis and clinical manifestations (36). Recently, in vitro work from several groups has suggested a putative link between type I IFNs, vascular damage, and atherosclerosis progression in SLE (4-6). However, whether these effects contribute significantly to endothelial dysfunction in vivo had not been determined.

Our observations suggest that type I IFNs have notable pleiotropic effects on the vasculature, from early EC damage and aberrant vascular repair, to plaque development and destabilization, followed by development of ACS through thrombosis enhancement. We show that type I IFNs play a direct, important role in CV damage and endothelial dysfunction development in vivo in lupus-prone mice and atheroma-prone mice, as well as in mouse strains not typically associated with lupus or enhanced atherothrombotic risk.

The nomenclature for vascular progenitor cells is undergoing constant revision. For the purposes of this work, we continue to refer to these cells as EPCs. IFN $\alpha$ is toxic to BM and blood EPCs in vitro $(4,8)$, and impairments in their phenotype and function may pro- 
mote the endothelial dysfunction that has been reported in murine and human lupus $(10,13)$. While endothelial function and EPC numbers are reduced and functionally impaired in chronic kidney disease (37), the modulation of endothelial and EPC function by type I IFNs appears to be independent of renal dysfunction or of other immune abnormalities characteristic of SLE. Indeed, male NZM mice, spared from overt lupus development and glomerulonephritis, still exhibit significant differences in vascular parameters when compared to male INZM mice. This is consistent with the results of previous studies of human EPCs/circulating angiogenic cells, where lupus disease activity did not explain the profound vascular phenotypic and functional abnormalities (4). These observations support a specific role for type I IFNs in endothelial dysfunction and loss of EPC numbers and function in vivo.

Our findings indicate that chronic and enhanced exposure to type I IFNs may be needed to induce significant decreases in EPC numbers in vivo, since acute exposure to IFN $\alpha$ did not lead to alterations in these parameters, although it significantly impaired EPC differentiation. It is also possible that these effects on the vasculature are mediated, at least in part, by other IFNs besides IFN $\alpha$, since the knockout system used in this study deleted signaling by all type I IFNs $(14,15)$. Indeed, while the differential effects that various type I IFNs play in the vasculature and in endothelial repair remain unclear, recent evidence suggests a role for type I IFNs other than IFN $\alpha$ in antiangiogenic responses and atherosclerosis modulation $(7,38)$.

Type I IFNs can impair the ability of stem cells to repopulate the $\mathrm{BM}$ niche. Therefore, the negative impact of IFNs on EPC function could indicate a more general stem cell impairment (39). Furthermore, type I IFNs can induce apoptosis and senescence of mature ECs (40). Therefore, long-term exposure to type I IFNs, as is observed in SLE, could promote sustained vascular damage and reduced local repair, leading to enhanced EPC and mature EC consumption, as shown in atherosclerosis models (33). Impaired EPC differentiation by type I IFNs may be associated with induction of antiangiogenic signatures in these cells and in other tissue, as we previously described in human lupus in vivo (8). This could create a cytokine profile that enhances antiangiogenic responses, promotes vasculopathy, and accelerates atherosclerosis.

Lupus-prone mice are resistant to florid dietinduced atherosclerosis, unless crossed with proatherosclerotic models (41-44), or used to generate chimeras following lupus BM transplantation into atherosclerosisprone mice (45). While those studies demonstrated that lupus immune dysregulation accelerates plaque development, they have not addressed the specific roles of type I IFNs and have been performed primarily in murine models that do not appear to depend on these cytokines for disease progression $(46,47)$.

Recent evidence points to a potentially crucial role for type I IFNs in atherosclerosis due to the ability of IFN $\alpha$ to increase foam cell formation (5), its increased expression in areas of unstable human arterial plaques (48), and the capacity of myeloid $\operatorname{IFN} \beta$ to worsen atherosclerosis (7). Strong associations of type I IFN signatures in human SLE with impaired peripheral arterial tone (49) and carotid intima-media thickness have been found (50). In the present study, we present direct in vivo evidence that type I IFNs significantly modulate plaque development and the severity of arterial macrophage and $\mathrm{T}$ cell infiltration in atherosclerosisprone murine systems. Under proatherogenic conditions, monocytes migrate to vessel walls and differentiate into macrophages that can become lipid-laden foam cells. The biologic properties of atherosclerotic plaque macrophages and $\mathrm{T}$ lymphocytes determine lesion size, composition, and stability $(45,51)$. Decreased macrophage infiltration in apoE ${ }^{-1-} \mathrm{IFNAR}^{-1-}$ mice may be secondary to impaired monocyte recruitment and/or differentiation into foam cells, as supported by recent observations $(7,52)$.

The proatherogenic effects of type I IFNs may be partially mediated by deleterious modulation of lipoproteins. While HDL is atheroprotective through roles in reverse cholesterol transport and antiinflammatory properties, it may become proinflammatory and promote atherothrombosis (26). SLE patients have increased proinflammatory HDL $(34,35)$, an abnormality we have now observed in female NZM mice. While oxidized HDL levels were decreased in female INZM mice, we could not elucidate if this was due to abrogation of type I IFN signaling on lipoprotein oxidation, or related to lack of lupus development in these mice. Since male NZM mice do not develop increased oxidized HDL, we used these mice as a reliable comparison. Furthermore, $\operatorname{AdIFN} \alpha$ administration did not increase HDL oxidation, even if it exacerbated lupus. It is possible that chronic, rather than acute, differences in type I IFN signaling are required for lipoprotein dysregulation. Nevertheless, our results suggest a putative role of these cytokines in lipoprotein oxidation and the redox environment in vivo, which requires further investigation.

While platelets are activated in SLE and display evidence of increased exposure to type I IFNs (6), the functional relevance of this phenomenon in vivo had 
not previously been addressed. In our study, NZM and apoE ${ }^{-/-}$mice exposed to IFN $\alpha$ had a shorter time to occlusive thrombosis upon photochemical injury. This was associated with enhanced platelet activation, independent of a lupus-prone genetic background. Since circulating activated platelets can exacerbate atherosclerosis in murine systems (53), it is possible that this type I IFN-induced phenomenon also contributed to plaque formation. Future studies should address how type I IFNs modulate endothelial and platelet activation in autoimmune and nonautoimmune backgrounds, and the interplay of these molecules with lipoproteins and other factors. Furthermore, our results shed light on previous reports that infections may precede or be associated with enhanced atherothrombotic risk (54,55). Indeed, this may be mediated via type I IFNs, following exposure to microbial products (48).

The results of the present study also support the notion that treatments disrupting type I IFN signaling, which are currently being tested in various autoimmune diseases, could promote additional benefits by hampering CV risk. Therefore, it will be important to include biomarkers of vascular damage and functional studies of endothelial health as end points of efficacy analyses for patients receiving these agents. Whether inhibition of these pathways in the general population would also decrease atherosclerosis progression should be investigated.

\section{ACKNOWLEDGMENTS}

We thank Dr. Chaim Jacob for providing breeding pairs of NZM2328 and NZM2328 IFN $\alpha \beta \mathrm{R}^{-1-}$ mice and Dr. Herbert W. Virgin for providing breeding pairs of IFN $\alpha \beta \mathrm{R}^{-/-}$ mice.

\section{AUTHOR CONTRIBUTIONS}

All authors were involved in drafting the article or revising it critically for important intellectual content, and all authors approved the final version to be published. Dr. Kaplan had full access to all of the data in the study and takes responsibility for the integrity of the data and the accuracy of the data analysis.

Study conception and design. Thacker, Koch, Pennathur, Davidson, Eitzman, Kaplan.

Acquisition of data. Thacker, Zhao, Smith, Luo, Wang, VivekanandanGiri, Rabquer, Pennathur.

Analysis and interpretation of data. Thacker, Smith, Luo, Vivekanandan-Giri, Rabquer, Koch, Pennathur, Eitzman, Kaplan.

\section{REFERENCES}

1. Cervera R, Khamashta MA, Font J, Sebastiani GD, Gil A, Lavilla $\mathrm{P}$, et al. Morbidity and mortality in systemic lupus erythematosus during a 10-year period: a comparison of early and late manifes- tations in a cohort of 1,000 patients. Medicine (Baltimore) 2003; 82:299-308.

2. Esdaile JM, Abrahamowicz M, Grodzicky T, Li Y, Panaritis C, du Berger R, et al. Traditional Framingham risk factors fail to fully account for accelerated atherosclerosis in systemic lupus erythematosus. Arthritis Rheum 2001;44:2331-7.

3. Manzi S, Meilahn EN, Rairie JE, Conte CG, Medsger TA Jr, Jansen-McWilliams L, et al. Age-specific incidence rates of myocardial infarction and angina in women with systemic lupus erythematosus: comparison with the Framingham Study. Am J Epidemiol 1997;145:408-15.

4. Denny MF, Thacker S, Mehta H, Somers EC, Dodick T, Barrat FJ, et al. Interferon- $\alpha$ promotes abnormal vasculogenesis in lupus: a potential pathway for premature atherosclerosis. Blood 2007;110: 2907-15.

5. Li J, Fu Q, Cui H, Ou B, Pan W, Shen N, et al. Interferon- $\alpha$ priming promotes lipid uptake and macrophage-derived foam cell formation: a novel link between interferon- $\alpha$ and atherosclerosis in lupus. Arthritis Rheum 2011;63:492-502.

6. Lood C, Amisten S, Gullstrand B, Jonsen A, Allhorn M, Truedsson L, et al. Platelet transcriptional profile and protein expression in patients with systemic lupus erythematosus: upregulation of the type I interferon system is strongly associated with vascular disease. Blood 2010;116:1951-7.

7. Goossens P, Gijbels MJ, Zernecke A, Eijgelaar W, Vergouwe MN, van der Made I, et al. Myeloid type I interferon signaling promotes atherosclerosis by stimulating macrophage recruitment to lesions. Cell Metab 2010;12:142-53.

8. Thacker SG, Berthier CC, Mattinzoli D, Rastaldi MP, Kretzler M, Kaplan MJ. The detrimental effects of IFN- $\alpha$ on vasculogenesis in lupus are mediated by repression of IL-1 pathways: potential role in atherogenesis and renal vascular rarefaction. J Immunol 2010; 185:4457-69.

9. Banchereau J, Pascual V. Type I interferon in systemic lupus erythematosus and other autoimmune diseases. Immunity 2006; 25:383-92.

10. Rajagopalan S, Somers EC, Brook RD, Kehrer C, Pfenninger D, Lewis E, et al. Endothelial cell apoptosis in systemic lupus erythematosus: a common pathway for abnormal vascular function and thrombosis propensity. Blood 2004;103:3677-83.

11. Anderson TJ, Gerhard MD, Meredith IT, Charbonneau F, Delagrange D, Creager MA, et al. Systemic nature of endothelial dysfunction in atherosclerosis. Am J Cardiol 1995;75:71B-4B.

12. Hsueh W, Abel ED, Breslow JL, Maeda N, Davis RC, Fisher EA, et al. Recipes for creating animal models of diabetic cardiovascular disease. Circ Res 2007;100:1415-27.

13. Thacker SG, Duquaine D, Park J, Kaplan MJ. Lupus-prone New Zealand Black/New Zealand White F1 mice display endothelial dysfunction and abnormal phenotype and function of endothelial progenitor cells. Lupus 2010;19:288-99.

14. Huang S, Hendriks W, Althage A, Hemmi S, Bluethmann H, Kamijo R, et al. Immune response in mice that lack the interferon- $\gamma$ receptor. Science 1993;259:1742-5.

15. Muller U, Steinhoff U, Reis LF, Hemmi S, Pavlovic J, Zinkernagel RM, et al. Functional role of type I and type II interferons in antiviral defense. Science 1994;264:1918-21.

16. Ramanujam M, Kahn P, Huang W, Tao H, Madaio MP, Factor $\mathrm{SM}$, et al. Interferon- $\alpha$ treatment of female $(\mathrm{NZW} \times \mathrm{BXSB}) \mathrm{F}_{1}$ mice mimics some but not all features associated with the Yaa mutation. Arthritis Rheum 2009;60:1096-101.

17. Nacionales DC, Kelly-Scumpia KM, Lee PY, Weinstein JS, Lyons $\mathrm{R}$, Sobel E, et al. Deficiency of the type I interferon receptor protects mice from experimental lupus. Arthritis Rheum 2007;56: 3770-83.

18. Zhao W, Thacker SG, Hodgin JB, Zhang H, Wang JH, Park JL, et al. The peroxisome proliferator-activated receptor $\gamma$ agonist 
pioglitazone improves cardiometabolic risk and renal inflammation in murine lupus. J Immunol 2009;183:2729-40.

19. Chakroborty D, Chowdhury UR, Sarkar C, Baral R, Dasgupta PS, Basu S. Dopamine regulates endothelial progenitor cell mobilization from mouse bone marrow in tumor vascularization. J Clin Invest 2008;118:1380-9.

20. Silverman MD, Haas CS, Rad AM, Arbab AS, Koch AE. The role of vascular cell adhesion molecule 1 / very late activation antigen 4 in endothelial progenitor cell recruitment to rheumatoid arthritis synovium. Arthritis Rheum 2007;56:1817-26.

21. Rabquer BJ, Amin MA, Teegala N, Shaheen MK, Tsou PS, Ruth $\mathrm{JH}$, et al. Junctional adhesion molecule-C is a soluble mediator of angiogenesis. J Immunol 2010;185:1777-85.

22. Amin MA, Volpert OV, Woods JM, Kumar P, Harlow LA, Koch AE. Migration inhibitory factor mediates angiogenesis via mitogen-activated protein kinase and phosphatidylinositol kinase. Circ Res 2003;93:321-9.

23. Eitzman DT, Westrick RJ, Nabel EG, Ginsburg D. Plasminogen activator inhibitor-1 and vitronectin promote vascular thrombosis in mice. Blood 2000;95:577-80.

24. Bodary PF, Gu S, Shen Y, Hasty AH, Buckler JM, Eitzman DT. Recombinant leptin promotes atherosclerosis and thrombosis in apolipoprotein E-deficient mice. Arterioscler Thromb Vasc Biol 2005;25:e119-22.

25. McPherson PA, Young IS, McKibben B, McEneny J. High density lipoprotein subfractions: isolation, composition, and their duplicitous role in oxidation. J Lipid Res 2007;48:86-95.

26. Pennathur S, Bergt C, Shao B, Byun J, Kassim SY, Singh P, et al. Human atherosclerotic intima and blood of patients with established coronary artery disease contain high density lipoprotein damaged by reactive nitrogen species. J Biol Chem 2004;279: 42977-83.

27. Vivekanandan-Giri A, Byun J, Pennathur S. Quantitative analysis of amino Acid oxidation markers by tandem mass spectrometry. Methods Enzymol 2011;491:73-89.

28. Waters ST, Fu SM, Gaskin F, Deshmukh US, Sung SS, Kannapell CC, et al. NZM2328: a new mouse model of systemic lupus erythematosus with unique genetic susceptibility loci. Clin Immunol 2001;100:372-83

29. Malyszko J. Mechanism of endothelial dysfunction in chronic kidney disease. Clin Chim Acta 2010;411:1412-20.

30. Coccia EM, Severa M, Giacomini E, Monneron D, Remoli ME, Julkunen I, et al. Viral infection and Toll-like receptor agonists induce a differential expression of type $\mathrm{I}$ and $\lambda$ interferons in human plasmacytoid and monocyte-derived dendritic cells. Eur J Immunol 2004;34:796-805.

31. Bengtsson AA, Sturfelt G, Truedsson L, Blomberg J, Alm G, Vallin $\mathrm{H}$, et al. Activation of type I interferon system in systemic lupus erythematosus correlates with disease activity but not with antiretroviral antibodies. Lupus 2000;9:664-71.

32. Bentzon JF, Falk E. Atherosclerotic lesions in mouse and man: is it the same disease? Curr Opin Lipidol 2010;21:434-40.

33. Zhu S, Liu X, Li Y, Goldschmidt-Clermont PJ, Dong C. Aging in the atherosclerosis milieu may accelerate the consumption of bone marrow endothelial progenitor cells. Arterioscler Thromb Vasc Biol 2007;27:113-9.

34. McMahon M, Grossman J, FitzGerald J, Dahlin-Lee E, Wallace DJ, Thong BY, et al. Proinflammatory high-density lipoprotein as a biomarker for atherosclerosis in patients with systemic lupus erythematosus and rheumatoid arthritis. Arthritis Rheum 2006;54: $2541-9$.

35. McMahon M, Grossman J, Skaggs B, FitzGerald J, Sahakian L, Ragavendra N, et al. Dysfunctional proinflammatory high-density lipoproteins confer increased risk of atherosclerosis in women with systemic lupus erythematosus. Arthritis Rheum 2009;60:2428-37.

36. Crow MK. Interferon- $\alpha$ : a therapeutic target in systemic lupus erythematosus. Rheum Dis Clin North Am 2010;36:173-86.
37. Bahlmann FH, Speer T, Fliser D. Endothelial progenitor cells in chronic kidney disease. Nephrol Dial Transplant 2010;25:341-6.

38. Taylor KL, Leaman DW, Grane R, Mechti N, Borden EC, Lindner DJ. Identification of interferon- $\beta$-stimulated genes that inhibit angiogenesis in vitro. J Interferon Cytokine Res 2008;28:733-40.

39. Sato T, Onai N, Yoshihara H, Arai F, Suda T, Ohteki T. Interferon regulatory factor-2 protects quiescent hematopoietic stem cells from type I interferon-dependent exhaustion. Nat Med 2009;15: 696-700.

40. Kaiser WJ, Kaufman JL, Offermann MK. IFN- $\alpha$ sensitizes human umbilical vein endothelial cells to apoptosis induced by doublestranded RNA. J Immunol 2004;172:1699-710.

41. Aprahamian T, Rifkin I, Bonegio R, Hugel B, Freyssinet JM, Sato $\mathrm{K}$, et al. Impaired clearance of apoptotic cells promotes synergy between atherogenesis and autoimmune disease. J Exp Med 2004;199:1121-31.

42. Feng X, Li H, Rumbin AA, Wang X, La Cava A, Brechtelsbauer $\mathrm{K}$, et al. $\mathrm{ApoE}^{-1-} \mathrm{Fas}^{-1-} \mathrm{C} 57 \mathrm{BL} / 6$ mice: a novel murine model simultaneously exhibits lupus nephritis, atherosclerosis, and osteopenia. J Lipid Res 2007;48:794-805.

43. Ma Z, Choudhury A, Kang SA, Monestier M, Cohen PL, Eisenberg RA. Accelerated atherosclerosis in ApoE deficient lupus mouse models. Clin Immunol 2008;127:168-75.

44. Stanic AK, Stein CM, Morgan AC, Fazio S, Linton MF, Wakeland EK, et al. Immune dysregulation accelerates atherosclerosis and modulates plaque composition in systemic lupus erythematosus. Proc Natl Acad Sci U S A 2006;103:7018-23.

45. Gautier EL, Huby T, Ouzilleau B, Doucet C, Saint-Charles F, Gremy G, et al. Enhanced immune system activation and arterial inflammation accelerates atherosclerosis in lupus-prone mice. Arterioscler Thromb Vasc Biol 2007;27:1625-31.

46. Hron JD, Peng SL. Type I IFN protects against murine lupus. J Immunol 2004;173:2134-42.

47. Schwarting A, Paul K, Tschirner S, Menke J, Hansen T, Brenner $\mathrm{W}$, et al. Interferon- $\beta$ : a therapeutic for autoimmune lupus in MRL-Fas $^{\mathrm{lpr}}$ mice. J Am Soc Nephrol 2005;16:3264-72.

48. Niessner A, Shin MS, Pryshchep O, Goronzy JJ, Chaikof EL, Weyand CM. Synergistic proinflammatory effects of the antiviral cytokine interferon- $\alpha$ and Toll-like receptor 4 ligands in the atherosclerotic plaque. Circulation 2007;116:2043-52.

49. Lee PY, Li Y, Richards HB, Chan FS, Zhuang H, Narain S, et al. Type I interferon as a novel risk factor for endothelial progenitor cell depletion and endothelial dysfunction in systemic lupus erythematosus. Arthritis Rheum 2007:56:3759-69.

50. Somers EC, Zhao W, Lewis EE, Wang L, Wing JJ, Sundaram B, et al. Type I interferons are associated with subclinical markers of cardiovascular disease in a cohort of systemic lupus erythematosus patients. PLoS One 2012;7:e37000.

51. Ye D, Zhao Y, Hildebrand RB, Singaraja RR, Hayden MR, Van Berkel TJ, et al. The dynamics of macrophage infiltration into the arterial wall during atherosclerotic lesion development in lowdensity lipoprotein receptor knockout mice. Am J Pathol 2011; 178:413-22.

52. Lee PY, Li Y, Kumagai Y, Xu Y, Weinstein JS, Kellner ES, et al. Type I interferon modulates monocyte recruitment and maturation in chronic inflammation. Am J Pathol 2009;175:2023-33.

53. Huo Y, Schober A, Forlow SB, Smith DF, Hyman MC, Jung S, et al. Circulating activated platelets exacerbate atherosclerosis in mice deficient in apolipoprotein E. Nat Med 2003;9:61-7.

54. Zurru MC, Alonzo C, Brescacin L, Romano M, Camera LA Waisman G, et al. Recent respiratory infection predicts atherothrombotic stroke: case-control study in a Buenos Aires healthcare system. Stroke 2009;40:1986-90.

55. Smeeth L, Thomas SL, Hall AJ, Hubbard R, Farrington P, Vallance P. Risk of myocardial infarction and stroke after acute infection or vaccination. N Engl J Med 2004;351:2611-8. 